RUNNING HEAD: Size and density mediate species interactions

\title{
Size and density mediate transitions between competition and facilitation
}

Hayley Cameron ${ }^{1}$, Tim Coulson ${ }^{2}$ and Dustin J. Marshall ${ }^{1}$

${ }^{1}$ Centre of Geometric Biology, School of Biological Sciences, Monash University, Melbourne, Victoria 3800, Australia.

${ }^{2}$ Department of Zoology, University of Oxford, Oxford, OX1 3PS, UK

*Corresponding author: Mailing address: School of Biological Sciences, Monash University, Clayton 3800; Phone number: +61 039905 5658; Email: Hayley.cameron@monash.edu

Co-authors email: Tim.coulson@zoo.ox.ac.uk,Dustin.marshall@monash.edu

Statement of authorship: TC, HC and DJM designed the experiment, HC conducted the experiments, TC and $\mathrm{HC}$ performed the analyses, $\mathrm{HC}$ and DJM wrote the first draft of the manuscript and all authors contributed to revisions.

Data accessibility statement: Data available from the Dryad Digital Repository: https://doi.org/10.5061/dryad.4p9t6v7

Keywords: competitive hierarchy, life history, integral projection model, population dynamics, response surface, interspecific interactions, trait variation 


\begin{abstract}
Species simultaneously compete with and facilitate one another. Size can mediate transitions along this competition-facilitation continuum, but the consequences for demography are unclear. We orthogonally manipulated the size of a focal species, and the size and density of a heterospecific neighbour, in the field using a model marine system. We then parameterised a size-structured population model with our experimental data. We found that heterospecific size and density interactively altered the population dynamics of the focal species. Size determined whether heterospecifics facilitated (when small) or competed with (when large) the focal species, while density strengthened these interactions. These sizemediated interactions also altered the pace of the focal's life history. We provide the first demonstration that size and density mediate competition and facilitation from a population dynamical perspective. We suspect such effects are ubiquitous, but currently underappreciated. We reiterate classic cautions against inferences about competitive hierarchies made in the absence of size-specific data.
\end{abstract}




\section{Introduction}

Species that use the same resources were traditionally viewed as competitors, but it is increasingly apparent that facilitation is also common - some species enhance the performance of others (Bertness \& Callaway 1994; Bruno et al. 2003). It is now clear that competition and facilitation occur simultaneously, such that the net outcomes of interactions represent a continuum - from strongly negative (competition) to strongly positive (facilitation). Both competition and facilitation influence species ranges (Bulleri et al. 2016), population and community dynamics (Butterfield 2009) and species coexistence (Buss \& Jackson 1979; Chesson 2000; Gross 2008; Johnson \& Bronstein 2019). Determining the factors that mediate competition and facilitation is therefore a priority in ecology.

A long-held view is that the size of organisms can influence the outcomes of species interactions (Buss 1980; Damuth 1981; Russ 1982; Weiner 1990). Size determines resource acquisition and consumption, so it is perhaps unsurprising that size mediates competition. For example, larger individuals both require more resources, and are better at appropriating resources, thus they often outcompete smaller organisms (Weiner 1990). On the other hand, smaller individuals have relatively lower resource requirements, such that at times, they may have a competitive advantage (Persson 1985; De Roos et al. 2003; Reuman et al. 2014).

Increasingly, it appears that size not only influences competition, but also facilitation (Lin et al. 2012; Lin et al. 2016; Malanson \& Resler 2016; Zepeda \& Martorell 2019). For instance, large herbivorous mammals facilitate smaller herbivores by removing grasses that would otherwise prevent these smaller species from accessing their target resources (VeseyFitzGerald 1960; Gordon 1988). In plants, the relative influence of facilitation and competition can depend on size and age (Miriti 2006; Schiffers \& Tielbörger 2006; ReismanBerman 2007; le Roux et al. 2013). Theory predicts that size-mediated shifts in competition and facilitation may be important for the dynamics and size-structure of populations (Chu et 
al. 2008; Lin et al. 2012; Lin et al. 2016), as well as species coexistence (Ranjan \& Bagchi 2016). Empirical demonstrations are rare, however, such that the consequences of sizemediated interactions remain unclear.

A key impediment to understanding size-mediated interactions is that empirical studies struggle to provide information at the appropriate scale (Hart et al. 2018). Empirical approaches typically examine the outcomes of interactions at the level of individuals, while theory considers the consequences for populations - specifically, whether interactions ultimately lead to coexistence or competitive exclusion. Crucially then, theory defines the 'winners' of species interactions across multiple generations (Hart et al. 2018). Under this definition, a number of limitations associated with classic studies emerge.

Most empirical approaches (including earlier work of our own: Cameron and Marshall 2019) use snapshot observations or short-term performance data to make extrapolations about size and species interactions (Buss 1980; Russ 1982). These classic studies in particular were undoubtedly foundational for demonstrating that size alters species interactions, but population-level inferences based on snapshot data can be deeply misleading (Fowler et al. 2006; Trinder et al. 2013; Hart et al. 2016). This dissonance stems in part from the fact that competitive ability (formally defined) comprises an organism's ability to: 1 . appropriate resources and supress other individuals (competitive effect); 2. withstand competition from neighbours (competitive response); and 3. grow in the absence of neighbours (intrinsic rate of increase - a population-level parameter; Hart et al. 2018). To illustrate, if Species A invariably overgrows Species B in a snapshot study, then traditionally A would be considered the superior competitor. But if B has a higher intrinsic rate of increase, then perhaps counter-intuitively, B will competitively exclude A over time (Hart et al. 2018). 
Size-mediated interactions complicate things further, such that snapshot approaches become even less informative. For example, imagine that Species A when large, negatively affects the performance of younger, smaller, individuals of Species B, but that the reverse is true when B is larger than A - snapshot approaches might conclude that competition is intransitive (Buss 1980; Russ 1982). However, as Buss (1980) presciently suggested, the ultimate outcome will then depend on the size-frequency distribution of the population. From our example above, large individuals of Species B may be so rare in the population that ultimately, Species A will outcompete B. Ontogenetic shifts in competition and facilitation complicate things further, such that net outcomes cannot be intuited. A rare demographic example found adult plants that received facilitation as juveniles contributed little to the overall dynamics of the population relative to isolated plants (Miriti et al. 2001; Miriti 2006). Thus, facilitation of individuals during any one life-stage may not be important at the population-level when integrated over the life cycle. Clearly then, an approach that integrates individual-level data across different performance metrics (mortality, growth and fecundity), and ontogenetic stages, is required to reliably predict the population-level outcomes of sizemediated interactions.

Demographic models provide one such approach. These models integrate empirically estimated demographic rates (survival, growth, reproduction) through time (i.e. ontogeny) to make projections about future population growth $(\lambda)$ under current ecological conditions (Caswell 2006). Demographic models can be directly parameterised with experimental data collected over the lifetime of organisms, and used to quantify the nature of species interactions at the population-level by comparing projections of $\lambda$ across experimental conditions (e.g. McPeek \& Peckarsky 1998; Bassar et al. 2015). In essence, demographic models provide the formal theoretical bridge between short-term estimates of individual-level performance and population-level outcomes. 
Integral Projection Models (IPMs) are one such model that investigates the dynamics of populations structured by continuous characters - typically body size (Easterling et al. 2000; Coulson et al. 2010; Ellner et al. 2016). These models are amenable to parameterisation with experimental data collected using designs common to studies of species interactions, such as response surfaces (Bassar et al. 2016). Response surface designs estimate interaction coefficients across a range of densities to investigate the nature of species interactions under realistic conditions (Inouye 2001). As such, both IPMs and response surfaces are powerful, but underused, tools for determining the factors that mediate transitions between competition and facilitation at the population-level.

Here, we test for size-mediated transitions in competition and facilitation by parameterising a single-species IPM with data from a manipulative field experiment. We use a marine invertebrate system that has provided some of the foundations of population ecology and species interactions (Buss \& Jackson 1979; Buss 1980; Russ 1982; Hart \& Marshall 2013), and have attributes particularly amenable to demographic studies in the field. We elaborate on traditional response surface experiments to create a trait-specific response 'cube' (Fig. 1), where we orthogonally varied the size of a focal species, as well as the size and density of a heterospecific species. The inclusion of density was particularly important given that facilitation may alter the relationship between size and density in non-intuitive ways (Chu et al. 2008). We monitored key demographic rates (survival, growth and reproduction) of the focal species across their lifetime under field conditions. We then integrated across these lifetime measures of performance using our IPM approach. We provide novel evidence that the size and density of a heterospecific species can mediate transitions along the competition-facilitation continuum to affect the population dynamics of another species.

\section{Methods}


Study system

We use two common filter-feeding bryozoans, Bugula neritina (hereafter Bugula) and Watersipora subtorquata (hereafter Watersipora), as our heterospecific and focal species, respectively. Both species are colonial, but differ in growth form: Watersipora is encrusting and grows by adding zooids (modular subunits) to the edges of the colony; Bugula is arborescent and buds new zooids from the tips of the colony branches (Fig. S1). Traditional views of competition predict that these species compete via different strategies: Watersipora should pre-empt space and over-grow other organisms; Bugula should exploit resources more efficiently (Jackson 1977). Both species are common to early-successional assemblages worldwide and primarily compete for space, food and oxygen (Hart et al. 2012; Hart \& Marshall 2013; Svensson \& Marshall 2015; Lagos et al. 2017), but interactions may switch from competitive to facilitative with increasing environmental stress (Hart \& Marshall 2013).

\section{Experimental methods}

To test for size-mediated interactions, we use a trait-specific, response cube that orthogonally varied the size of our focal species (Watersipora), as well as the size and density of our heterospecific species (Bugula; Fig. 1). For Waterispora, we used a continuous range of size-classes (as colony area) that spanned 0.185 to $414.2 \mathrm{~cm}^{2}$. For Bugula, we had five size-classes based on the number of times the colony had branched (1, 2, 3, 4 and 5 bifurcations). We had six heterospecific densities $\left(0,1,2,3,5\right.$ and 10 colonies per $121 \mathrm{~cm}^{2}$;) reflecting natural densities of Bugula in the field (Hart \& Marshall 2013). Our manipulations of heterospecific size and density generated several biomass isoclines - whereby the total number of zooids were equivalent, but differentially 'packaged', as either many small, or a few large, heterospecifics (Fig. 1c). The experiment included 30 combinations of competitor 
sizes and densities, and each combination received eight focal colonies (replicates) that spanned the distribution of Watersipora sizes (see Table S1).

We performed the experiment at the Blairgowrie Yacht Squadron, Victoria, Australia (38²1'20.2"S, 144²4'22.8"E) from December 2013 to April 2014. For our manipulations, we obtained colonies of both species that varied in size and attached them to PVC plates (11 x $11 \mathrm{~cm}$ ) using standard methods (Hart et al. 2012; see also Appendix S1). We deployed the plates (experimental units) into the field by attaching them to the undersides of PVC backing panels that hung $1 \mathrm{~m}$ below the water surface. In total, our experiment included 1080 colonies of known size of both species, distributed across 240 plates and 15 backing panels (Appendix S1). We measured key demographic rates for 240 focal Watersipora colonies.

We monitored the performance of our focal colonies from photographs taken every two weeks for a total of eight weeks. We consider performance at the level of the colony (genet) rather than individual zooids (ramets), given that colony size alters demographic rates (growth, survivorship and fecundity) and drives population dynamics in modular organisms (Caswell 1985). We scored colonies as alive if they were present on the plates and contained living zooids, while we scored the colonies as dead if they were missing from the plates or all the zooids had died. We measured the growth of the colonies as their total area $\left(\mathrm{cm}^{2}\right.$; Image J) at each census. We measured fecundity as the total number of embryos brooded by the colony. We note that all focal colonies were pre-reproductive at the beginning of the experiment and we first detected reproduction 6 weeks after our manipulations. Thus, focal colonies were able to adjust their reproductive schedules in response to the heterospecific environment.

We noted changes in the density of heterospecifics (Bugula) by monitoring their survival at each census (Appendix S2). We randomised the position of the plates within backing panels and removed any non-experimental settlers (both our study species and other 
organisms) every two weeks to avoid exogenous competition. Recruitment onto the plates was extremely low during the experiment - thus, other species did not affect the interactions we observe and model. We stopped the experiment after 8 weeks in the field because by 10 weeks, most of the heterospecific colonies (Bugula) had died. Mass die-offs are common for Bugula populations in our study region, and typically coincide with seasonal changes in the environment at the end of summer (Marshall 2005), as we also observed.

\section{Modelling framework}

To explore how the size and density of heterospecifics (Bugula) affect the population dynamics of the focal species (Watersipora), we integrated across our various performance measures (vital rates) using an Integral Projection Model (IPM; Easterling et al. (2000)). Our IPM is both a size- and age-structured model (Coulson et al. 2010):

$$
\begin{gathered}
n\left(z^{\prime}, 1, t+1\right)=\sum_{a} \int\left[D\left(z^{\prime} \mid z, a, y_{b}, N_{b}\right) M\left(z, a, y_{b}, N_{b}\right) B\left(z, a, y_{b}, N_{b}\right) n(z, a, t)\right] d z \\
n\left(z^{\prime}, a+1, t+1\right)=\int\left[G\left(z^{\prime} \mid z, a, y_{b}, N_{b}\right) S\left(z, a, y_{b}, N_{b}\right) n(z, a, t)\right] d z
\end{gathered}
$$

where the continuous trait for size, $z$, is the square root of colony area $\left(\mathrm{cm}^{2}\right)$. In our model, the first equation deals with settlers, the second equation deals with the survival and growth of colonies. Our model is thus multigenerational - individuals reproduce and recruit into the populations but we do not model the dispersal of offspring outside their natal environment, nor their planktonic mortality.

$\mathrm{D}\left(z^{\prime} \mid \mathrm{z}, y_{b}, a, N_{b}\right)$ is the conditional probability density function that describes the distribution of offspring sizes, $z^{\prime}$, produced by parental colonies of size $z$ at time $t+1$. $a$ is the experimental age class, $y_{b}$ is the size of the competitor (as bifurcations), and $N_{b}$ is the density of the competitor. $M\left(z, a, y_{b}, N_{b}\right)$ is a continuous function describing the mean number of 
offspring produced by an individual with the colony area $z$, and of experimental age $a$, given the size and density of the competitor. $S\left(z, a, y_{b}, N_{b}\right)$ and $B\left(z, a, y_{b}, N_{b}\right)$ are continuous functions that describe the probability of an individual with colony area $z$, and experimental age $a$, at the beginning of the interval surviving and reproducing at the end of the interval, respectively, as a function of competitor density and size. $G\left(z^{\prime} \mid z, a, y_{b}, N_{b}\right)$ is the Gaussian probability density function describing transitions from colony area $z$ at time $t$ to colony area $z^{\prime}$ at time $t+1$ among survivors as a function of experimental age, competitor size and density. $n(z, t)$ is the distribution of Watersiporia size at time $t$ such that $N(t)=$ $\int_{x}^{y} n(z, t) d z$ is the number of individuals between size $z$ and $y$. We note that, $y_{b}$ and $N_{b}$ are experimental factors that we manipulated and are not dynamic in the model, but heterospecific colonies obviously grew and died throughout the experiment (Appendix S2). While we do not model these dynamics explicitly, they are implicit in our phenomenological projections of focal population growth rate based on our experimental data.

For a comprehensive description of the model and the underlying vital rate functions, see Appendices S3 and S4, respectively. From this model, we then calculated a number of quantities:

1. The asymptotic population growth rate $(\lambda)$ for Watersipora across the range of heterospecific densities and sizes we used in our experiments.

2. The elasticity of $\lambda$ to each matrix element $\alpha_{i, j}: \frac{\partial \log (\lambda)}{\partial \log \left(\alpha_{i, j}\right)}$. This quantity is a partial derivative that describes the proportional change in the population growth rate to a proportional change in the matrix element. We summed the elasticities of $\lambda$ for all matrix elements across all focal sizes in the first-age class to ask under which heterospecific environments are smaller, younger individuals most important to 
population growth. A large value of elasticity means that individuals in the first age class have a large impact on the dynamics of the focal species.

3. The reproductive value distribution $(v)$ across the range of heterospecific sizes and densities used in our experiments. Reproductive values describe the expected numerical representation of the descendants of an individual of a given size and age within the population at time $t$ at some arbitrary time point far in the future. We report the reproductive values of small (0.63), medium (14.3) and large (27.94) Watersipora colonies at experimental age one, but we note that we obtain equivalent results at later ages.

As per standard practice, we calculated the above-mentioned quantities at equilibrium - that is, the model had reached a stable population structure and constant population growth rate (Caswell 2006). We note this definition of equilibrium does not imply that our model (or empirical manipulations) had reached a constant population size, nor that the communities in which our species exist were at equilibrium.

\section{Results}

Heterospecific size, density and biomass affect the population growth of the focal species

We found that the size and density of a heterospecific species (Bugula) interact to regulate the vital rates (see Appendix S4 for details) and population dynamics of a focal species (Watersipora). Importantly, heterospecific size mediated the transition between competition and facilitation, while heterospecific density largely determined the strength of these interactions (Fig. 2a). The change in $\lambda$ across heterospecific regimes show these transitions along the competition-facilitation continuum - competition occurs when values of $\lambda$ within a given heterospecific environment are lower than under heterospecific-free conditions (when heterospecific density is 0 ), while the inverse indicates facilitation. 
The population growth rate of Watersipora increased with increasing densities of smaller Bugula relative to heterospecific-free conditions. In particular, $\lambda$ was highest when heterospecifics were small and numerous - indicating strong facilitation. These interactions transitioned from facilitative to increasingly competitive as the size of Bugula increased. Importantly, when Bugula were largest and most dense, $\lambda$ was $<1$ - indicative of competitive exclusion. Overall, we found a $70 \%$ difference in estimates of $\lambda$ between Watersipora populations that were surrounded by many, smaller heterospecifics (i.e. when $\lambda$ was highest) and many, larger heterospecifics (i.e. $\lambda$ was lowest). Interestingly, Bugula of intermediate size did not alter the population growth rate of Watersipora relative to heterospecific-free conditions - that is, intermediate Bugula had consistently neutral effects, regardless of their density.

Heterospecific size and density were stronger determinants of interspecific interactions than total heterospecific biomass across much of the parameter space (demonstrated by the superimposed biomass isoclines in Fig 2a). For example, when total heterospecific biomass was $<200$ zooids, interactions changed from mildly competitive at lower densities of large heterospecifics, to facilitative at higher densities of smaller heterospecifics. Thus, the interactive effects of heterospecific size and density decouple the relationship between population growth and total heterospecific biomass.

Elasticities revealed that when Watersipora could persist in the presence of Bugula (i.e. $\lambda>1$ ), smaller colonies in the first age class had the greatest contribution to our projections of $\lambda$ (Fig. 2b). Indeed, these smaller, younger individuals contributed most to $\lambda$ when heterospecifics were small and numerous (i.e. regions of facilitation), or large and few (i.e. regions of mild competition). In contrast, these younger, smaller colonies were least important to population growth at high densities of larger heterospecifics (i.e. regions of intense competition). 
Heterospecific size, density and biomass affect the size-specific reproductive values of the focal species

The size, density and total biomass of heterospecifics had complex, non-linear effects on the size-specific reproductive values $(v)$ of the focal species. In heterospecific-free conditions (when heterospecific biomass is 0 ), reproductive values increased with colony size, as shown by the upward shift in intercept moving from the left to right panels in Fig. 3. In contrast, the presence of heterospecifics altered these size-specific reproductive values relative to heterospecific-free conditions (i.e. in regions of both facilitation and competition; compare Fig 2a to Fig 3a-c).

For the smallest and largest Watersipora colonies, total heterospecific biomass (rather than size and density) determined reproductive values, albeit in opposing directions. Unsurprisingly, very small Watersipora colonies had relatively small $v$, but within this size class, $v$ was surprisingly highest at the largest heterospecific biomasses (Fig. 3a \& d). Note this increase came not from greater growth of Watersipora under increasing biomass, but because reproduction was induced at a smaller size (see Appendix S4). In contrast, for the largest Watersipora colonies, $v$ was maximised at lower heterospecific biomasses (Fig. 3c \& f).

For intermediate Watersipora, complex interactions between heterospecific size, number and biomass altered $v$ (Fig $3 \mathrm{~b} \& \mathrm{e}$ ). Broadly, $v$ increased with heterospecific biomass to a point, but then declined again at very high biomasses. Additionally, while intermediate biomasses had consistent effects on $v$ for intermediate Watersipora, the way that biomass was partitioned into the size and number of individuals altered $v$ when total biomass was very low or very high. When biomass was low, $v$ was highest when biomass comprised fewer, large heterospecifics relative to many, smaller heterospecifcs. Conversely, when heterospecific 
biomass was very high, $v$ peaked at intermediate densities of large heterospecifics, but declined as these densities increased (Fig $3 b$ \& e).

To summarise then, when competition is most intense (i.e. higher densities of larger heterospecifics; see Fig. 2a) small and medium individuals contribute the most offspring to future population growth, while few large individuals reproduce (Fig. 3; see also Appendix S4). In contrast, when facilitation dominates (see Fig 2a), small and intermediate individuals appear to delay reproduction, such that only the largest individuals contribute offspring (Fig. 3; see also Appendix S4).

\section{Discussion}

We demonstrate that heterospecific size and density mediates transitions between facilitation and competition and alters the population dynamics of a focal species. Interspecific interactions shifted from facilitative when heterospecifics were small, to increasingly competitive when heterospecifics were large. Meanwhile, increasing heterospecific density strengthened these interactions - that is, size-mediated competition and facilitation were density-dependent. Importantly, these size-mediated effects were not transient; rather they persisted across the life cycle of the focal species (c.f. Miriti et al. 2001). We also found that heterospecific size and density had complex, non-linear effects on the size-specific reproductive values of the focal species, suggesting that size-mediated competition and facilitation can alter the pace of the life history. Other examples of complex interactions between the size and density of organisms in driving competition and facilitation are rare, but we suspect they are likely ubiquitous.

\section{Consequences for populations}

The total biomass of a given population should determine its resource use, and therefore competitive effects, on others (Damuth 1981). In our study, heterospecific size and 
density had interactive effects on the population-dynamics of the focal species - thus heterospecific biomass was a poor predictor of the focal species' response to interspecific interactions (see Fig. 2). Our findings therefore contradict classic theory that did not consider the role of positive interactions in regulating populations (Damuth 1981). Recent studies propose different pathways through which facilitation may decouple traditional biomassdensity relationships (Chu et al. 2008; Ghedini et al. 2017). Our findings are congruent with these ideas, although explicit tests are required to identify the mechanisms via which facilitation disrupts classic assumptions of size-mediated resource use.

One explanation for our results is that heterospecific size and density alters the availability of resources for the focal species. Arborescent Bugula colonies (our heterospecific species) may alter water-flows and the delivery of water-borne resources to conspecific neighbours - leading to intraspecific facilitation and competition (Cameron et al. 2016; Cameron \& Marshall 2019; see also Thompson et al. 2015 for studies using structural mimics). We suspect similar mechanisms determine interspecific interactions between Bugula and Watersipora. For example, smaller Bugula facilitated Watersipora and these interactions were density-dependent. Importantly then, it appears that a larger number of heterospecifics are required to ameliorate conditions for the focal species, as observed in other systems (Leslie 2005; Bishop et al. 2012; Svanfeldt et al. 2017). That Watersipora benefits from amelioration of flow and resource stress also has empirical support - reduced flows enhance feeding rates of encrusting byrozoans in the laboratory, as well as their performance in the field (Okamura 1985; Svanfeldt et al. 2017). Furthermore, amelioration may be particularly important here, given the high flow rates at our site (Lagos et al. 2017).

As heterospecific size increased, however, interspecific interactions became increasingly competitive, and again, were density dependent. Competition likely increased with heterospecific size because of the relatively higher resource demands of larger 
individuals (Schoener 1983; Weiner 1990; in Bugula: Barneche et al. 2017). Overall then, our results suggest that many smaller Bugula disrupt water flow but do not consume all the resources they generate, therefore they facilitate Watersipora. In contrast, many large Bugula may increase resource supply, but also consume much of these resources (Cameron \& Marshall 2019), thus they compete with Watersipora. Nevertheless, we acknowledge that other, less likely, forms of habitat amelioration (e.g. predator defence) may have contributed to our findings. An important next step, therefore, would be to estimate resource consumption by our focal species under various heterospecific regimes and realistic flow conditions (e.g. Ghedini et al. 2017). Such experiments would determine whether size-mediated resource amelioration and consumption drives facilitation and competition in our system.

Population-level consequences of size-based shifts in facilitation and competition are unlikely to be restricted to our study system. Rather, we suspect such interactions are widespread, but have previously gone undetected due to the rarity of studies that use population dynamical approaches. For example, facilitation occurs via resource amelioration in a range of systems, and in some cases is size-dependent (e.g. in mammals: VeseyFitzGerald 1960; Gordon 1988; other aquatic systems: Cardinale et al. 2002; Whalen \& Stachowicz 2017; birds: Russell et al. 2007; and plants: Pretzsch et al. 2013). Size and density may also determine interactions when facilitation occurs via non-resource related forms of amelioration. Certainly, general models of amelioration predict size-based shifts between facilitation and competition (Lin et al. 2012; Lin et al. 2016; Malanson \& Resler 2016). Our study thus highlights the importance of viewing size-mediated interactions from a population dynamical perspective, and we encourage further tests to determine their generality and identify their underlying mechanisms.

Consequences for life histories 
Changes in the size-specific reproductive values that we observe suggest the focal species shifted its life history in response to the heterospecific environment. For example, smaller focal colonies contributed the most descendants to future population growth (i.e. had highest reproductive values) when competition was highest (i.e. high heterospecific biomasses) - indicative of an acceleration in the life history. Early reproduction can occur when competition reduces the probability of future growth and(or) survival in other systems, including other colonial marine invertebrates (Harvell \& Grosberg 1988; Kozlowski \& Weiner 1997; Hesse et al. 2008). As far as we are aware, our study is the first to demonstrate these effects in response to the size of neighbours, but size-mediated competition can alter life histories within species (Bassar et al. 2016). Recall, however, that higher densities of larger Bugula competitively excluded Watersipora. At the population-level, therefore, shifts towards earlier reproduction did not compensate for the negative effects of competition. Indeed, elasticities reveal that small, young (reproductive) colonies are relatively unimportant for population growth when competition is most intense.

Much theory has examined the role of competition in shaping life histories (e.g. Kozlowski \& Weiner 1997; Bassar et al. 2016), while the role of facilitation has traditionally been ignored, yet can be equally strong (Zepeda \& Martorell 2019). We found that facilitation slowed the focal's life history relative to heterospecific-free conditions. In regions of facilitation, larger individuals contributed the most descendants to future population growth (i.e. had higher reproductive values) because focal colonies delayed reproduction and invested more in growth, such that they eventually reproduced more as larger colonies (Appendix S4: Table S1). Elasticities further support this interpretation - smaller, younger (non-reproductive) colonies were important for population growth in regions of facilitation. As such, facilitation on early survival and growth persists at the population-level because a higher number of younger individuals transitioned to later stages and (eventually) reproduced 
more as larger colonies (Appendix S4: Table S1) - positively affecting population growth (c.f. Miriti et al. 2001). Crucially, we show that facilitation, not just competition, may shape life-history strategies and this warrants further exploration.

\section{Consequences for coexistence}

Classic studies presciently suggested that size promotes competitive intransitivities that promote coexistence among species (Buss 1980; Russ 1982). While such studies were undoubtedly foundational, they could not access the population dynamical consequences of size-mediated interactions (Hart et al. 2018). We show that size-mediated interactions can generate profound differences in population dynamics $(\sim 70 \%$ difference in population growth) that not only mediate competitive hierarchies, but also change the direction of species interactions. Given these findings, studies that aim to identify competitive hierarchies should use a size-based approach, integrated into a dynamic framework. To illustrate, our current findings contradict previous studies in our system that did not manipulate body size, but rather estimated interactions across a restricted size range (Hart et al. 2012; Hart \& Marshall 2013). In these earlier studies, Bugula had, at most, weak competitive effects on Watersipora. In contrast, we find strong effects that depend on Bugula sizes and densities. Such idiosyncrasies may explain why average competitive effects appear weak when sizestructure is ignored, and may lead to misleading conclusions about putative competitors.

Our findings highlight two important points. First, the overall outcome of species interactions will depend on the size-structure of the interacting species within a given community (Buss 1980), which often differ in time and space (including in our species: Marshall 2005). Certainly, different population structures yield vastly different population dynamical outcomes within species (Miriti et al. 2001; Bassar et al. 2015). Second, recall that we found complex, nonlinear effects of heterospecific size, density and biomass on both the 
population growth, and size-specific reproductive values, of the focal species. Thus, examining performance for any one size combination of heterospecific and focal (even the most common sizes) will provide inaccurate estimates of species interactions due to nonlinear averaging (i.e. Jensen's inequality: Jensen 1906). Jensen's inequality thus precludes meaningful inferences based on the average sizes of interacting species, and can have important implications for species coexistence (Bolnick et al. 2011; Hart et al. 2016). As such, we suggest an explicit partitioning of size-specific effects is necessary to fully capture the dynamics, and identify the ultimate outcomes, of species interactions.

Our findings may have further applications for theories of coexistence. Cohorts of Bugula will alternatively facilitate and compete with Watersipora. How this alternation of benefits and costs ultimately affect coexistence is unclear. Recent studies proposed that opposite-signed interactions may represent an equalising mechanism for species coexistence (Zepeda \& Martorell 2019) - our finding of size-mediated competition and facilitation may have similar consequences. Importantly, current theory largely focuses on size differences among species. We show intraspecific size variation among heterospecifics simultaneously determines competition and facilitation - an alternative pathway through which species using the same resources may coexist (Gross 2008). Such theory is yet to consider body size in mediating these effects, but our results suggest this exciting possibility.

\section{Acknowledgements}

We are grateful to the MEEG lab for assistance in the field and laboratory. We thank G. Ghedini and M. Alvarez Noriega for providing feedback on earlier drafts. DJM and TC were supported by the Australian Research Council. The thoughtful comments of four anonymous reviewers greatly improved our manuscript and clarified our arguments about the necessity of population models for understanding sized-mediated species interactions. 


\section{Literature Cited}

Barneche, D.R., White, C.R. \& Marshall, D.J. (2017). Temperature effects on mass-scaling exponents in colonial animals: a manipulative test. Ecology, 98, 103-111.

Bassar, R.D., Childs, D.Z., Rees, M., Tuljapurkar, S., Reznick, D.N. \& Coulson, T. (2016). The effects of asymmetric competition on the life history of Trinidadian guppies. Ecol. Lett., 19, 268-278.

Bassar, R.D., Heatherly, T., Marshall, M.C., Thomas, S.A., Flecker, A.S. \& Reznick, D.N. (2015). Population size-structure-dependent fitness and ecosystem consequences in Trinidadian guppies. J. Anim. Ecol., 84, 955-968.

Bertness, M.D. \& Callaway, R. (1994). Positive interactions in communities. Trends Ecol. Evol., 9, 191193.

Bishop, M.J., Byers, J.E., Marcek, B.J. \& Gribben, P.E. (2012). Density-dependent facilitation cascades determine epifaunal community structure in temperate Australian mangroves. Ecology, 93, 1388-1401.

Bolnick, D.I., Amarasekare, P., Araújo, M.S., Bürger, R., Levine, J.M., Novak, M. et al. (2011). Why intraspecific trait variation matters in community ecology. Trends Ecol. Evol., 26, 183-192.

Bruno, J.F., Stachowicz, J.J. \& Bertness, M.D. (2003). Inclusion of facilitation into ecological theory. Trends Ecol. Evol., 18, 119-125.

Bulleri, F., Bruno, J.F., Silliman, B.R. \& Stachowicz, J.J. (2016). Facilitation and the niche: implications for coexistence, range shifts and ecosystem functioning. Funct. Ecol., 30, 70-78.

Buss, L.W. (1980). Competitive intransitivity and size-frequency distributions of interacting populations. Proc. Nat. Acad. Sci., 77, 5355-5359.

Buss, L.W. \& Jackson, J.B.C. (1979). Competitive networks: Nontransitive competitive relationships in cryptic coral reef environments. Am. Nat., 113, 223-234.

Butterfield, B.J. (2009). Effects of facilitation on community stability and dynamics: synthesis and future directions. J. Ecol., 97, 1192-1201.

Cameron, H. \& Marshall, D.J. (2019). Can competitive asymmetries maintain offspring size variation? A manipulative field test. Evolution, doi:10.1111/evo.13790.

Cameron, H., Monro, K., Malerba, M., Munch, S. \& Marshall, D. (2016). Why do larger mothers produce larger offspring? A test of classic theory. Ecology, 97, 3452-3459.

Cardinale, B.J., Palmer, M.A. \& Collins, S.L. (2002). Species diversity enhances ecosystem functioning through interspecific facilitation. Nature, 415, 426.

Caswell, H. (1985). The evolutionary demography of clonal reproduction. In: Population Biology and Evolution of Clonal Organisms (ed. J. B. C. Jackson, LWB, and R. E. Cook). Yale University Press New Haven, Connecticut, U.S.A., pp. 187-224.

Caswell, H. (2006). Matrix Population Models: Constructuion, Analysis and Interpretation. 2nd ed. Oxford University Press Inc, Oxford, UK.

Chesson, P. (2000). Mechanisms of Maintenance of Species Diversity. Ann. Rev. Ecol. Syst., 31, 343366.

Chu, C.-J., Maestre, F.T., Xiao, S., Weiner, J., Wang, Y.-S., Duan, Z.-H. et al. (2008). Balance between facilitation and resource competition determines biomass-density relationships in plant populations. Ecol. Lett., 11, 1189-1197.

Coulson, T., Tuljapurkar, S. \& Childs, D.Z. (2010). Using evolutionary demography to link life history theory, quantitative genetics and population ecology. J. Anim. Ecol., 79, 1226-1240.

Damuth, J. (1981). Population density and body size in mammals. Nature, 290, 699.

De Roos, A.M., Persson, L. \& McCauley, E. (2003). The influence of size-dependent life-history traits on the structure and dynamics of populations and communities. Ecol. Lett., 6, 473-487.

Easterling, M.R., Ellner, S.P. \& Dixon, P.M. (2000). Size-specific sensitivity: Applying a new structured populaton model. Ecology, 81, 694-708.

Ellner, S.P., Childs, D.Z. \& Rees, M. (2016). Data-driven modelling of structured populations: $A$ practical guide to the Integral Projection Model. Springer, New York. 
Fowler, N.L., Overath, R.D. \& Pease, C.M. (2006). Detection of density dependence requires density manipulations and calcuation of $\lambda$. Ecology, 87, 655-664.

Ghedini, G., White, C.R. \& Marshall, D.J. (2017). Does energy flux predict density-dependence? An empirical field test. Ecology, 98, 3316 - 3126.

Gordon, I.J. (1988). Facilitation of red deer grazing by cattle and its impact on red deer performance. J. Appl. Ecol., 25, 1-9.

Gross, K. (2008). Positive interactions among competitors can produce species-rich communities. Ecol. Lett., 11, 929-936.

Hart, S.P., Burgin, J.R. \& Marshall, D.J. (2012). Revisiting competition in a classic model system using formal links between theory and data. Ecology, 93, 2015-2022.

Hart, S.P., Freckleton, R.P. \& Levine, J.M. (2018). How to quantify competitive ability. J. Ecol., 106, 1902-1909.

Hart, S.P. \& Marshall, D.J. (2013). Environmental stress, facilitation, competition, and coexistence. Ecology, 94, 2719-2731.

Hart, S.P., Schreiber, S.J. \& Levine, J.M. (2016). How variation between individuals affects species coexistence. Ecol. Lett., 19, 825-838.

Harvell, C.D. \& Grosberg, R.K. (1988). The timing of sexual maturity in clonal animals. Ecology, 69, 1855-1864.

Hesse, E., Rees, M. \& Muller-Scharer, H. (2008). Life-history variation in contrasting habitats: Flowering decisions in a clonal perennial herb (Veratrum album). Am. Nat., 172, 196-E213.

Inouye, B.D. (2001). Response surface experimental designs for investigating interspecific competition. Ecology, 82, 2696-2706.

Jackson, J.B.C. (1977). Competition on marine hard substrata: The adaptive significance of solitary and colonial strategies. Am. Nat., 111, 743-767.

Jensen, J.L.W.V. (1906). Sur les fonctions convexes et les inegalites entre les valeurs moyennes. Acta Math., 30, 175-193.

Johnson, C.A. \& Bronstein, J.L. (2019). Coexistence and competitive exclusion in mutualism. Ecology, 100, e02708.

Kozlowski, J. \& Weiner, J. (1997). Interspecific allometries are by-products of body size optimization. Am. Nat., 149, 352-380.

Lagos, M.E., Barneche, D.R., White, C.R. \& Marshall, D.J. (2017). Do low oxygen environments facilitate marine invasions? Relative tolerance of native and invasive species to low oxygen conditions. Glob. Chang. Biol.,23, 2321-2330.

le Roux, P.C., Shaw, J.D. \& Chown, S.L. (2013). Ontogenetic shifts in plant interactions vary with environmental severity and affect population structure. New Phytol., 200, 241-250.

Leslie, H.M. (2005). Positive intraspecific effects trump negative effects in high-density barnacle aggregations. Ecology, 86, 2716-2725.

Lin, Y., Berger, U., Grimm, V. \& Ji, Q.-R. (2012). Differences between symmetric and asymmetric facilitation matter: exploring the interplay between modes of positive and negative plant interactions. J. Ecol., 100, 1482-1491.

Lin, Y., Berger, U., Yue, M. \& Grimm, V. (2016). Asymmetric facilitation can reduce size inequality in plant populations resulting in delayed density-dependent mortality. Oikos, 125, 1153-1161.

Malanson, G.P. \& Resler, L.M. (2016). A size-gradient hypothesis for alpine treeline ecotones. J Mt. Sci., 13, 1154-1161.

Marshall, D.J. (2005). Geographical variation in offspring size effects across generations. Oikos, 108, 602-608.

McPeek, M.A. \& Peckarsky, B.L. (1998). Life histories and the strengths of species interactions: Combining mortality, growth and fecundity effects. Ecology, 79, 867-879.

Miriti, M.N. (2006). Ontogenetic shift from facilitation to competition in a desert shrub. Journal of Ecology, 94, 973-979. 
Miriti, M.N., Joseph Wright, S. \& Howe, H.F. (2001). The effects of neighbors on the demography of a dominant desert shrub (Ambrosia Dumosa). Ecol. Monogr., 71, 491-509.

Okamura, B. (1985). The effects of ambient flow velocity, colony size, and upstream colonies on the feeding success of Bryozoa. II. Conopeum reticulum (Linnaeus), an encrusting species. J. Exp. Mar. Biol. Ecol., 89, 69-80.

Persson, L. (1985). Asymmetrical Competition: Are Larger Animals Competitively Superior? Am. Nat., 126, 261-266.

Pretzsch, H., Schütze, G. \& Uhl, E. (2013). Resistance of European tree species to drought stress in mixed versus pure forests: evidence of stress release by inter-specific facilitation. Plant Biol., $15,483-495$.

Ranjan, R. \& Bagchi, S. (2016). Functional response and body size in consumer-resource interactions: Unimodality favors facilitation. Theoret. Pop. Biol.,110, 25-35.

Reisman-Berman, O. (2007). Age-related change in canopy traits shifts conspecific facilitation to interference in a semi-arid shrubland. Ecography, 30, 459-470.

Reuman, D.C., Holt, R.D. \& Yvon-Durocher, G. (2014). A metabolic perspective on competition and body size reductions with warming. J. Anim. Ecol., 83, 59-69.

Russ, G.R. (1982). Overgrowth in a marine epifaunal community: Competitive hierarchies and competitive networks. Oecologia, 53, 12-19.

Russell, A.F., Langmore, N.E., Cockburn, A., Astheimer, L.B. \& Kilner, R.M. (2007). Reduced egg investment can conceal helper effects in cooperatively breeding birds. Science, 317, 941944.

Schiffers, K. \& Tielbörger, K. (2006). Ontogenetic shifts in interactions among annual plants. J. Ecol., 94, 336-341.

Schoener, T.W. (1983). Field experiments on interspecific fompetition. Am. Nat., 122, 240-285.

Svanfeldt, K., Monro, K. \& Marshall, D.J. (2017). Field manipulations of resources mediate the transition from intraspecific competition to facilitation. J. Anim. Ecol., 86, 654-661.

Svensson, J.R. \& Marshall, D.J. (2015). Limiting resources in sessile systems: food enhances diversity and growth of suspension feeders despite available space. Ecology, 96, 819-827.

Thompson, M.L., Marshall, D.J. \& Monro, K. (2015). Non-contact competition in a sessile marine invertebrate: causes and consequences. Mar. Ecol. Prog. Ser., 522, 115-125.

Trinder, C.J., Brooker, R.W. \& Robinson, D. (2013). Plant ecology's guilty little secret: understanding the dynamics of plant competition. Funct. Ecol., 27, 918-929.

Vesey-FitzGerald, D.F. (1960). Grazing succession among east african game animals. J.Mammal., 41, 161-172.

Weiner, J. (1990). Asymmetric competition in plant populations. Trends Ecol. Evol., 5, 360-364.

Whalen, M.A. \& Stachowicz, J.J. (2017). Suspension feeder diversity enhances community filtration rates in different flow environments. Mar. Ecol. Prog. Ser., 570, 1-13.

Zepeda, V. \& Martorell, C. (2019). Seed mass equalises the strength of positive and negative plantplant interactions in a semi-arid grassland. Oecologia. 

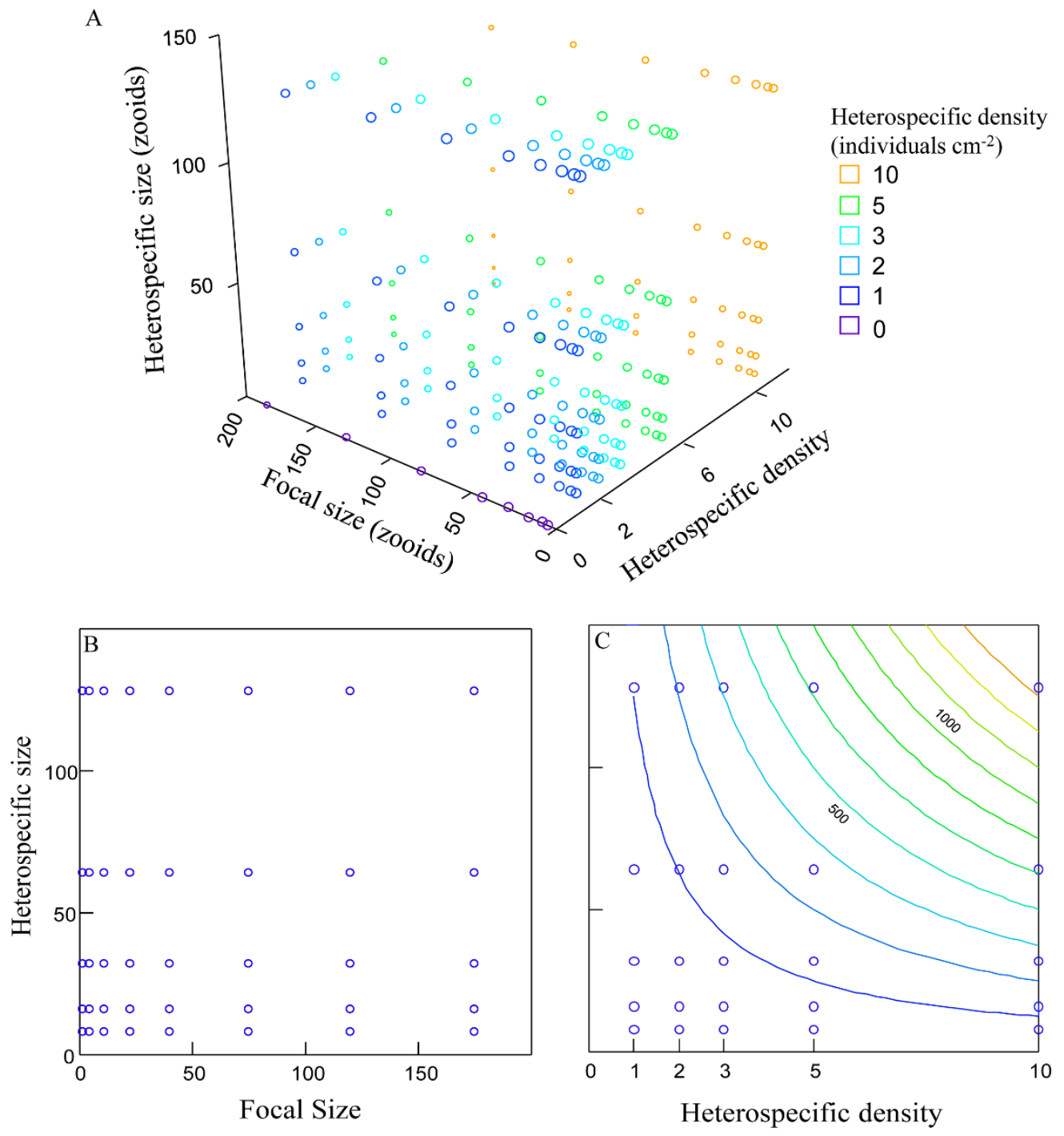

Figure 1. A. Schematic of the trait-specific, response cube used to test the effects of the size and densities (legend) of heterospecifics (Bugula) on the size-structured population dynamics of a focal species (Watersipora). B. Variation in the absolute and relative sizes (zooids) of the focal and heterospecific species (absolute size difference range of focal to heterospecific: 126 to 167 zooids; relative size ratio range of focal to heterospecific: 0.01 to 21.88 ). C. The heterospecific environments (Bugula sizes and densities) used to generate isoclines of heterospecific biomass (total zooids; coloured lines). Overall, the experiment included 30 heterospecific size and density combinations replicated across eight focal colonies of variable size, and we measured key demographic rates for 240 focal Watersipora colonies in the field across their lifetime. 

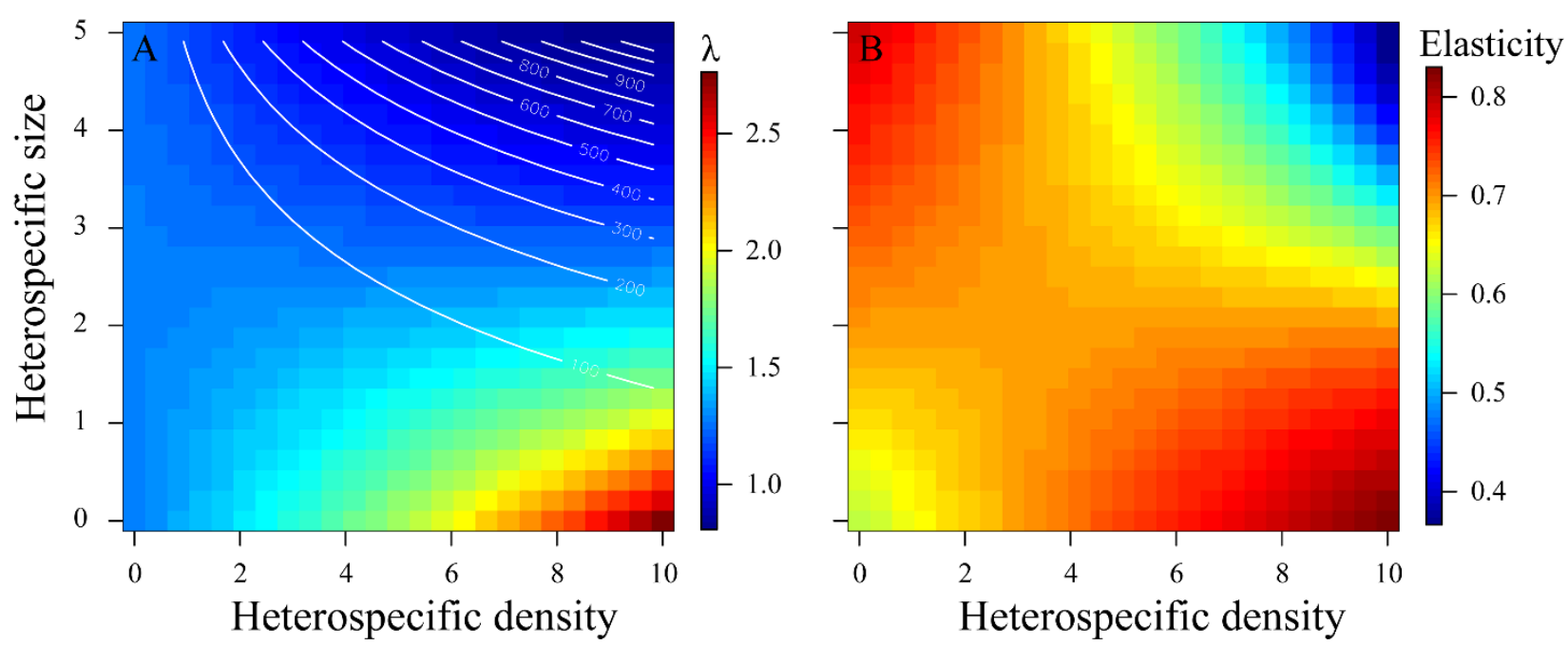

Figure 2. The effects of heterospecific (Bugula) size and density on: A. the projected population growth rate $(\lambda)$ of the focal species (Watersipora). Note that values of $\lambda$ greater than those in heterospecific-free conditions (when heterospecific density is 0 ) indicate facilitation, while values of $\lambda$ less than those in heterospecific-free conditions indicate competition. Values of $\lambda<1$ predicts competitive exclusion of the focal species. White contour lines show equivalencies in total heterospecific biomass (as zooids) - which are the product of heterospecific size and density (see also Fig. 1c). B. The summed elasticities for each demographic parameter (survival, growth and reproduction) for all Watersipora sizes in the first-age class. Larger values of elasticity indicate that smaller, younger colonies contribute relatively more to $\lambda$ than smaller elasticity values. 

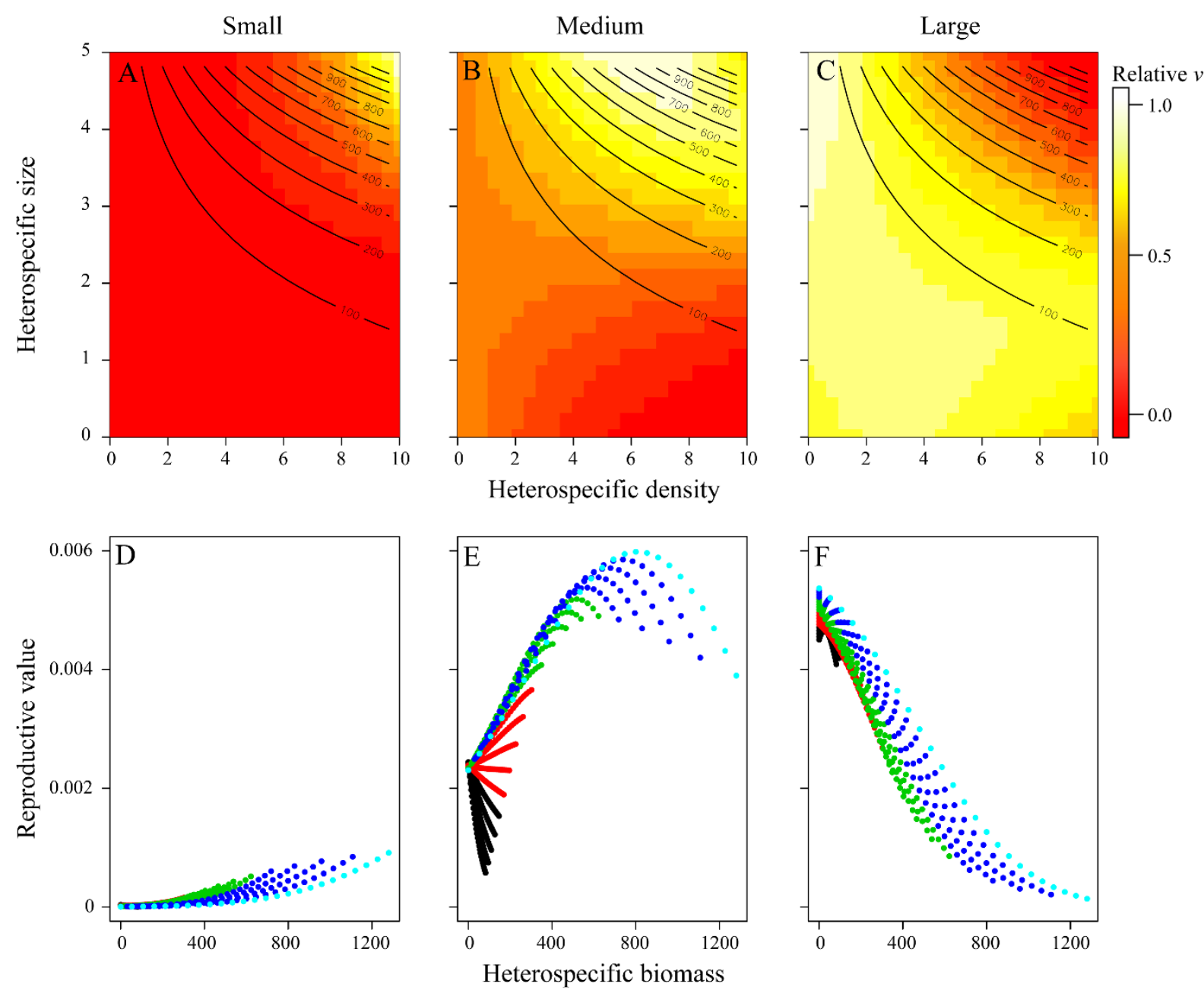

Figure 3. The reproductive values $(v)$ of small $(\mathbf{A}, \mathbf{D})$, intermediate $(\mathbf{B}, \mathbf{E})$ and large $(\mathbf{C}, \mathbf{F})$ Watersipora colonies in response to the size, density and total biomass of heterospecifics (Bugula). Panels A-C show relative values of $v$ within each Watersipora size class (legend) as a function of heterospecific size (as bifurcations) and density (individuals $121 \mathrm{~cm}^{-2}$ ). Black contour lines represent equivalencies of heterospecific biomass (as zooids) - i.e. the product of heterospecific size and density (see Fig 1c). Panels D-F are absolute values of $v$ plotted against total heterospecific biomass (zooids). Coloured dots represent the size-classes of the heterospecific (as bifurcations: black $=1$, red $=2$, green $=3$, navy $=4$, aqua $=5$ ). The intercepts for these panels represent reproductive values for small (D), intermediate (E) and large $(\mathbf{F})$ focal colonies under neighbour-free conditions. Note that in $\mathbf{D}$, the reproductive values of smaller heterospecifics (black and red lines) are zero. 